\title{
1200 Mirror Array Integrated with CMOS for Photonic Switching: Application of Mechanical Leveraging and Torsional Electrostatic Actuation To Reduce Drive Voltage Requirements and Increase Angular Tilt
}

\author{
James H. Smith, Steven S. Nasiri, Janusz Bryzek, Mitch Novack, James B. Starr, \\ Haesung Kwon, Anthony F. Flannery, David L. Marx, Zhenfang Chen, and Elaheh Sigari \\ Transparent Networks, Inc. \\ Santa Clara, CA 95050
}

\begin{abstract}
The design, fabrication, and performance of a 1200 mirror array for photonic switching applications are presented. Torsional electrostatic actuators are combined with angular amplifiers derived from 4-bar mechanisms to improve the mechanical performance of the devices to $8^{\circ}$ while maintaining the switched drive voltage requirements at $120 \mathrm{~V}$. The process technology and the integration of the mirror array with a custom drive ASIC is also discussed briefly. The mirrors are being used in a telecommunication application to form the basis of a 1200 port optical switch.
\end{abstract}

\section{INTRODUCTION}

The basic physics to overcome in electrostatic actuator design is the $2^{\text {nd }}$ order relationship between electric field (applied voltage divided by actuator gap) and the actuation force. So, to maintain force as a constant, the applied voltage must increase linearly with increases in the maximum displacement of the amplifier. In many applications, tens to hundreds of microns of motion are required. This requires very large gaps and subsequently hundreds of volts to drive these devices. A number of innovations have improved upon the basic performance of the parallel plate electrostatic drive including the comb drive [1] and the application of leverage to comb drives [2,3]. Leveraging has also been applied to improve the performance of mechanical sensors [4].

\section{MECHANICAL AND ELECTROSTATIC DESIGN}

The tradeoff between force and electrode spacing is a fundamental design issue in mirror arrays for optical switching applications. Since these mirrors are typically $0.5 \mathrm{~mm}$ to $2.0 \mathrm{~mm}$ in size, the spacing between the platform and the underlying electrode must be considerable to prevent mechanical interference of the platform and the electrode. Also, the parallel plate-like drives (as well as torsional drives) exhibit non-linear behaviors that are difficult to control beyond $\sim 30-40 \%$ of the initial gap. This results in gap spacings of 200 microns or more in a typical design. This large spacing drives the actuation voltage requirements to extremely high levels such as $350 \mathrm{~V}$ or more.

A concept similar to the mechanical leverage demonstrated in linear actuators can also be applied to achieve angular leverage. Figure 1 illustrates the classical 4-bar linkage mechanism. If the dimensions of the linkages are appropriately chosen, angular amplification can be readily achieved between the linkages $\left(\Delta \theta_{2}>\Delta \theta_{1}\right)$. With angular amplification, the gap spacing for torsional actuators can be reduced with a subsequent reduction in drive voltage. The classic 4-bar uses pin joints as the pivoting mechanism. To eliminate reliability issues and to enable fabrication, these pin joints are replaced with spring designs to form virtual pivots[5]. Additionally, the suspension of the mirror includes shock/overvoltage/overrange protection and electrostatic shielding.
The Transparent Networks Leveraged Virtual Pivot TM suspension, first disclosed by Nasiri [6], combines an angular amplifier with a virtual pivot point and torsional actuators to reduce drive voltage as well as improve the angular range of the mirror array. A leverage ratio of 4:1 (illustrated in Figure 2) has enabled the use of $120 \mathrm{~V}$ on the drive ASIC while achieving full angular performance of $8^{\circ}$. Although $120 \mathrm{~V}$ is higher than most CMOS, it is easily within the range of niche CMOS foundries who specialize in custom processes or by stacking of transistors in conventional CMOS foundries. The basic limit to switching voltages on a drive ASIC is the tub to substrate isolation breakdown voltage. This can range from $30 \mathrm{~V}$ to over $150 \mathrm{~V}$ depending upon the particular process. $120 \mathrm{~V}$ does not require exotic isolation methods such as dielectric isolation nor expensive materials such as SOI.

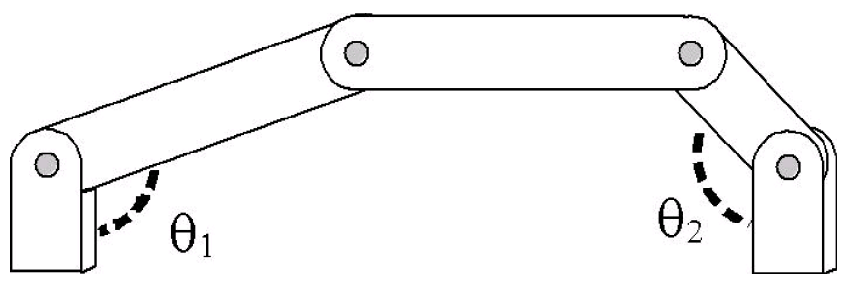

Figure 1. A basic 4-bar mechanism for mechanical angular amplification. With appropriate choice of geometry, the relationship between the changes in $\theta_{1}$ and $\theta_{2}$ can be used to amplify the motions at the respective pin joints.

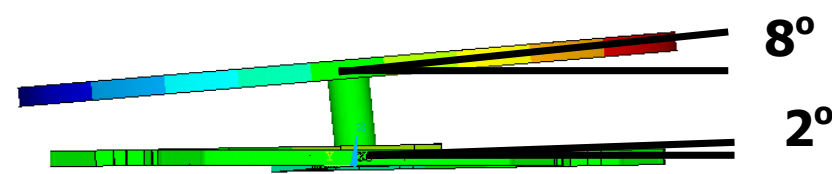

Figure 2. A compliant version of a 4-bar mechanism is used in the mechanical mirror design to amplify the angular motion movement of the actuators $\left(2^{\circ}\right)$ to achieve a larger angular tilt of the mirror's reflective platform $\left(8^{\circ}\right)$.

\section{FABRICATION}

The mirror fabrication is separated into CMOS fabrication, electrode fabrication, and MEMS fabrication. The MEMS fabrication has both a pre- and post-CMOS portion and includes 8 mask layers. First, CMOS is built in a foundry process. Next, the electrode assembly (a multi-level conductor/dielectric stack) is built on top of the foundry CMOS in a separate foundry operation. Both of these operations use standard foundry operations that support wafer flows in excess of 10,000 wafers per month. A MEMS wafer stack including the mirror and the suspension is prebuilt in an unreleased form from two fusion-bonded wafers using DRIE and wafer thinning. The pre-built mirrors, still in wafer form, are bonded using one of two low temperature bonding 
techniques (depending upon application) to the CMOS/electrode array. The completed wafer stack of 3 wafers is post-processed to thin the mirror platform, coat the mirrors with a reflective coating, and release the mirrors resulting in the mirror array shown in Figure 3. The optical coating of the mirror is gold with an adhesion layer / diffusion barrier. A cross-sectional SEM of the mechanical portion of the mirror array is shown in Figure 4.

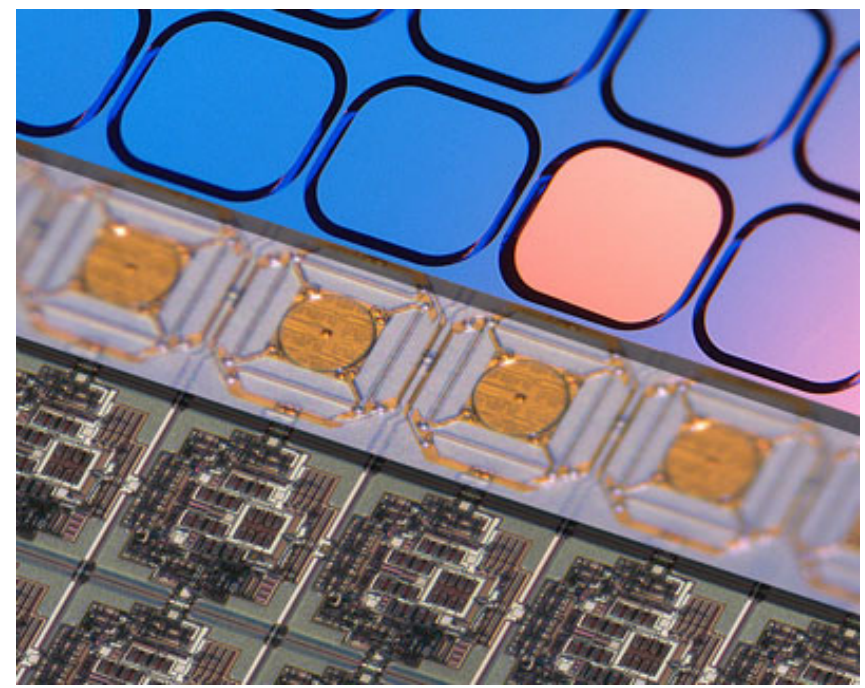

Figure 3. The three functional layers in the mirror device (CMOS, Electrode, and Mechanical) overlayed to show the structure of the mirror array. The bottom CMOS layer provides high voltage switching $(120 \mathrm{~V})$; the intermediate Electrode layer provides routing, shielding, and electrodes; the top Mechanical layer provides the optically-reflecting surface and mechanical suspension. For reference, the cell pitch in the photograph is approx. $1 \mathrm{~mm}$.

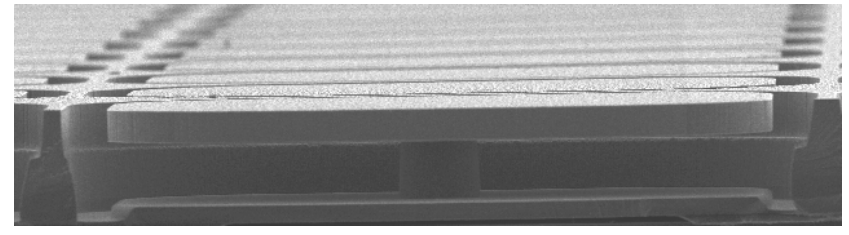

Figure 4. A cross-sectional SEM of a single cell in the MEMSportion of the mirror array.

\section{PERFORMANCE}

The demonstration of leveraged actuation through the static performance of the mirror is presented below and was obtained using external drive signals and a passive electrode array. Full dynamic performance of the mirror integrated with its drive ASIC and feedback loop has been demonstrated and will be reported in a subsequent paper.

Figure 5 illustrates the experimentally measured mirror tilt angle vs. drive voltage for one of the initial mirror designs. Simulations of subsequent designs show drive voltage requirements as low as $70 \mathrm{~V}$ (switched) with tilt angles of $10^{\circ}$ through optimization of suspension and electrode design. In all cases, a common negative bias equal to $2 / 3$ of the maximum switched voltage is applied to the MEMS actuator.

In its dynamic configuration within the optical switch, the mirror has demonstrated optical switch closure and stabilization in under $8 \mathrm{~ms}$ and an ability to handle $1 \mathrm{~W}$ of switched optical power.

\section{CONCLUSIONS}

The application of leveraged torsional electrostatic actuation to 3-D mirror arrays for photonic switching has been presented. The leveraged mechanical design drops drive voltage requirements to $120 \mathrm{~V}$ to achieve an $8^{\circ}$ tilt angle. The design of the mirror also hides the suspension beneath the reflecting platform and isolates the packaging and actuation stresses from the reflective platform. This results in extremely close packing density ( $90 \%$ linear fill) and large angular deflections $>8^{\circ}$ which aid the performance of high port count, low-loss optical switching systems.

A MEMS fabrication process has been demonstrated using 8 masks that is compatible with CMOS from commonly available foundries. This process uses a pre-built MEMS stack, wafer bonding to CMOS, and post-processing of the final 3-wafer stack to release the structures.

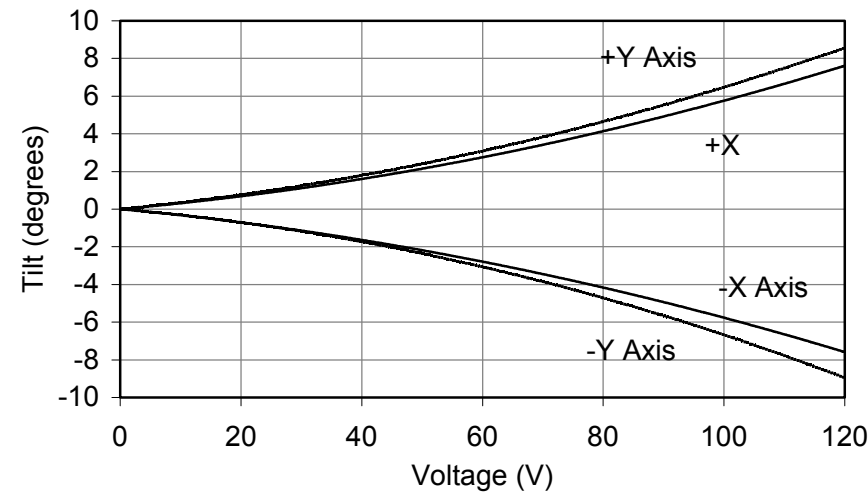

Figure 5. Static angle as a function of switched voltage for a mirror tilting on two orthogonal axes ( $X$ and $Y$ ). A constant mirror bias of $-90 \mathrm{~V}$ is used on the mirror array in addition to the switched voltage.

\section{REFERENCES}

1. W. C. Tang, T.-C. H. Nguyen, M. W. Judy, and R. T. Howe, "Electrostatic-comb drive of lateral polysilicon resonators," Sensors and Actuators, vol. A21-23, pp. 328-331. 1990.

2. J. Sniegowski and C. Smith, "An Application of Mechanical Leverage to Microactuation," 8th International Conference on Solid-State Sensors and Actuators, and Eurosensors IX, Proc. TRANSDUCERS '95/Eurosensors IX, Stockholm, Sweden, June 25-29, 1995, Vol. 2, pp. 364-367 (paper 325-PB9).

3. M. Rodgers, et al., "A New Class of High Force, Low-Voltage, Compliant Actuation Systems," Solid-State Sensor and Actuator Workshop, Hilton Head Island, South Carolina, June 4-8, 2000.

4. T. Roessig, R. Howe, A. Pisano, and J. Smith "SurfaceMicromachined Resonant Accelerometer," 1997 International Conference on Solid-State Sensors and Actuators, Chicago, IL, June 16-19, 1997, Vol. 2, pp. 859-862.

5. M. Murphy, A. Midha and L. Howell, "The topological synthesis of compliant mechanisms," Mechanism and Machine Theory, 31 (1996) 185-199.

6. S. Nasiri, "Microelectromechanical Mirror and Mirror Array," U.S. patent application 09/779189, Feb. 2001.

\section{ACKNOWLEDGEMENTS}

Special acknowledgement is deserved by J. Garate, M. Wang, D. Tactac, and P. Le for the fabrication of these devices. Optical photographs by R. Barnard. Many thanks to C. Ray, N. Maluf, and S. Terry for technical advice. CMOS design by H. Gurcan, D. Skurnik, and R. Sprague to be reported in future publications. 